Post-print version of the paper:

Zoltan Gingl, Peter Makra, and Robert Vajtai, Fluct. Noise Lett. 01, L181 (2001). ( ) World

Scientific Publishing Company.

DOI: 10.1142/S0219477501000408 (http://dx.doi.org/10.1142/S0219477501000408)

\title{
HIGH SIGNAL-TO-NOISE RATIO GAIN BY STOCHASTIC RESONANCE IN A
} DOUBLE WELL

\author{
ZOLTAN GINGL, PETER MAKRA and ROBERT VAJTAI \\ Department of Experimental Physics, University of Szeged \\ Dóm tér 9., Szeged, H-6720 Hungary \\ Email: gingl@physx.u-szeged.hu;phil@neptun.physx.u-szeged.hu;vajtai@physx.u-szeged.hu
}

Received 5 September 2001

Revised 24 September 2001

Accepted 25 September 2001

\begin{abstract}
We demonstrate that signal-to-noise ratio (SNR) can be significantly improved by stochastic resonance in a double well potential. The overdamped dynamical system was studied using mixed signal simulation techniques. The system was driven by wideband Gaussian white noise and a periodic pulse train with variable amplitude and duty cycle. Operating the system in the non-linear response range, we obtained SNR gains much greater than unity. In addition to the classical SNR definition, the ratio of the total power of the signal to the power of the noise part was also measured and it showed better signal improvement.
\end{abstract}

Keywords: Stochastic resonance; signal-to-noise ratio; double well potential.

\section{Introduction}

In certain systems, noise can optimize the signal transfer - that is, adding a given amount of noise to the input can maximize the signal-to-noise ratio (SNR) at the output. This phenomenon is called stochastic resonance (SR) and it is one of the most exciting topics in current noise research. SR has been observed in bistable and monostable dynamical systems, threshold devices, SQUIDs, lasers, etc (see [1-12] and references therein). In addition, SR is related to signal transfer in chemical and biological systems and is promising in neuron modeling based on noisy excitations [5, 7].

SR actually means that the output SNR $\left(S N R_{\text {out }}\right)$ has a maximum as a function of input noise intensity. In most cases, the input $S N R\left(S N R_{\text {in }}\right)$ exceeds $S N R_{\text {out }}$, yet the question arises whether it is possible to have larger SNR at the output than at the input - in other words, whether a noisy signal can get less noisy when transmitted through a stochastic resonator. After several unsuccessful attempts to answer this question, it was theoretically proved in $1995[13,14]$ that no SNR improvement is possible with a stochastic resonator working in the linear response (LR) range. LR means that the transfer of the signal component is linear even though the stochastic resonator is a strongly non-linear device; this phenomenon occurs when the signal amplitude is much smaller than the amplitude of the noise. Most of the earlier experiments and computer simulations were carried out under the LR condition in order to avoid creating higher harmonics in the periodic signal. We need not, however, confine our research to the LR limit because a wide range of signals, such as frequency-modulated, phase-modulated or pulse-code-modulated signals etc, are not destroyed by statically non-linear transfer (non-linearity without phase shift).

The aforesaid finding did not rule out SNR gain in the non-linear response (NLR) range, so subsequent studies assumed the condition of NLR. The first theoretical results 
proving that SR can yield significant SNR improvement were published in 1996 [1]. In this case, the system was a monostable threshold device, a level-crossing detector (LCD), and a model neural signal, a spike train with random initial times of spikes, served as an input. When operated in the strongly NLR limit, the system produced very high SNR gains far beyond any earlier expectation. Later a similarly large SNR gain was observed in numerical simulations with the same kind of LCD [2], but this time with a deterministic input signal, a periodic spike train. Similar results were obtained in saturating threshold stochastic resonator models [3]. In 2000, SNR gain was found in a simple bistable system, the Schmitt trigger [15].

In all the investigations mentioned above, the system that served as a stochastic resonator was non-dynamical. In this paper, we shall examine the possibility of SNR improvement in a dynamical SR system, the 'SR-classic' double well potential. Since it has recently been shown that if the periodic excitation is sinusoidal, the SNR gain is almost always below unity even though the system works in the NLR range [16], we shall use a symmetric periodic pulse train with variable amplitude and duty cycle as the input signal, to which a Gaussian white noise is added.

\section{Modeling SR in the Double Well Potential}

The system under scrutiny is a particle moving in a double well potential, excited by a periodic pulse train and a Gaussian white noise. The overdamped dynamics of the particle are given by the following Langevin equation:

$$
\frac{d x}{d t}=x-x^{3}+p(t)+w(t)
$$

where $p(t)$ is the periodic excitation and $w(t)$ represents a Gaussian white noise. In our simulations, the correlation time of the noise was always smaller than the width of the pulses in the deterministic excitation.

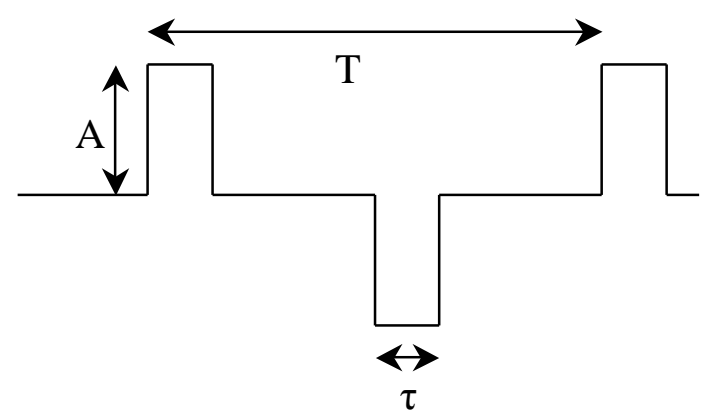

Fig. 1. The shape of the periodic input signal $p(t)$

The shape of the periodic signal is shown in Fig. 1, where $A$ denotes the amplitude, $T$ stands for the period, $\tau$ is the width of the pulses and the duty cycle of the signal is defined as $2 \tau / T$. The amplitude is expressed as a percentage of the threshold value (the amount of excitation needed for the particle to cross the potential barrier between the two potential wells when noise is not present). 
We have developed a mixed-signal — both analog and digital — system for our investigations. The noise and the periodic excitation were generated digitally and then converted to the analog domain using D/A converters. In order to solve the Langevin equation of the system with analog methods, we first transformed it into an integral equation:

$$
x(t)=\int_{0}^{t}\left[x\left(t^{\prime}\right)-x^{3}\left(t^{\prime}\right)+p\left(t^{\prime}\right)+w\left(t^{\prime}\right)\right] d t^{\prime} .
$$

The mathematical operations necessary to solve this integral equation, such as integration, multiplication, addition and subtraction, were realized by analog circuits. The whole system was driven by a high-performance digital signal processor (DSP) and the signals were measured by high-resolution A/D converters with fast roll-off anti-aliasing filters. A host personal computer running LabVIEW controlled the DSP and was also used to evaluate the measured data. The block diagram of the system is shown in Fig. 2.

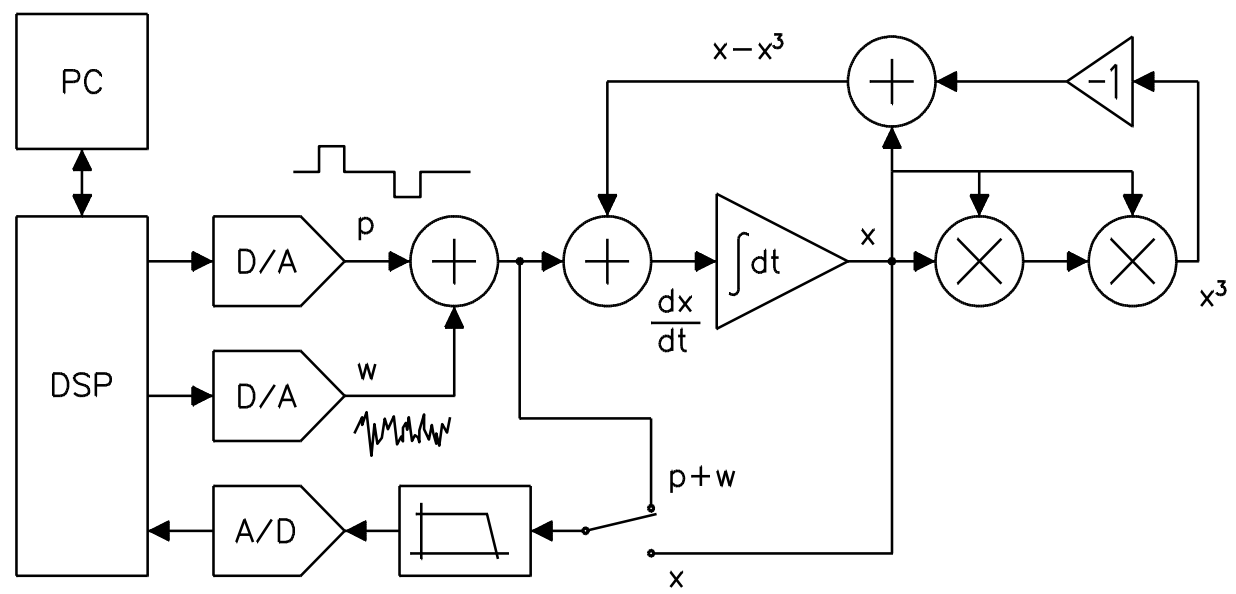

Fig. 2. Block diagram of the mixed-signal simulation system. The periodic and noisy signals are generated digitally and fed into an analog simulations system. The solution $x(t)$ is digitized by an $\mathrm{A} / \mathrm{D}$ converter and the data is processed by a DSP and the host PC.

One thousand samples, each consisting of 2048 data points, were measured and Fourier transformed to obtain the power spectral density, from which we calculated the value of the SNR. The output and input SNRs, $S N R_{\text {out }}$ and $S N R_{\text {in }}$, were measured by the same equipment and calculated exactly in the same way in order to avoid problems with the comparison - see the switch in front of the anti-aliasing filter connected to the A/D converter in Fig. 2. We used two SNR definitions: the classical narrowband definition most widely used in the field of SR [17]

$$
S N R=\frac{\lim _{\Delta f \rightarrow 0} \int_{f_{0}-\Delta f}^{f_{0}+\Delta f} S(f) d f}{S_{N}\left(f_{0}\right)},
$$


and a wideband SNR given by the ratio of the total power of the deterministic part of the signal $P_{S}$ to the total power of the noise part $P_{N}$ :

$$
S N R_{w}=\frac{P_{S}}{P_{N}}=\frac{\sum_{k=1}^{\infty} \lim _{\Delta f \rightarrow 0} \int_{k f_{0}-\Delta f}^{k f_{0}+\Delta f} S(f) d f}{\int_{0}^{\infty} S_{N}(f) d f} .
$$

In the definitions above, $f_{0}$ denotes the frequency of the deterministic signal, $S(f)$ stands for the power spectral density of the signal and $S_{N}(f)$ signifies the power spectral density of the noise component in the signal. Note here that the classical narrowband definition has long been perceived as insufficient to describe noisy signals correctly. Researchers have introduced several alternative methods for characterizing the quality of signal transfer, such as those based on entropy [14, 18] or channel capacity [19]. The wideband definition we suggest, $S N R_{w}$, is usually applied in practical measurements and it reflects the quality of the transfer better than the narrowband SNR. Figure 3 highlights this fact, where $S N R$ and $S N R_{w}$ is compared on a simple signal consisting of a sinusoidal plus noise. The $1 \mathrm{~V}$ amplitude sinusoidal with frequency of $30 \mathrm{~Hz}$ was added to a $16 \mathrm{kHz}$ bandwidth Gaussian white noise and this signal was filtered by a second order Butterworth bandpass filter with cutoff frequencies of $20 \mathrm{~Hz}$ and $40 \mathrm{~Hz}$. As one can expect, the noisy signal becomes much clearer after filtering, which is reflected only by $S N R_{w}, S N R$ is the same for both signals within the measurement error: SNR uses only a very small frequency range from the background noise around the signal frequency and the intensity of the noise in this range is altered by the same factor as that of the sinusoidal signal.

The SNR gain we are interested in is simply the ratio of the output SNR to the input SNR:

$$
G=\frac{S N R_{\text {out }}}{S N R_{\text {in }}},
$$

Of course, we also calculated a wideband gain from the wideband counterparts of $S N R_{\text {in }}$ and $S N R_{\text {out }}$ :

$$
G_{w}=\frac{S N R_{o u t, w}}{S N R_{i n, w}} .
$$



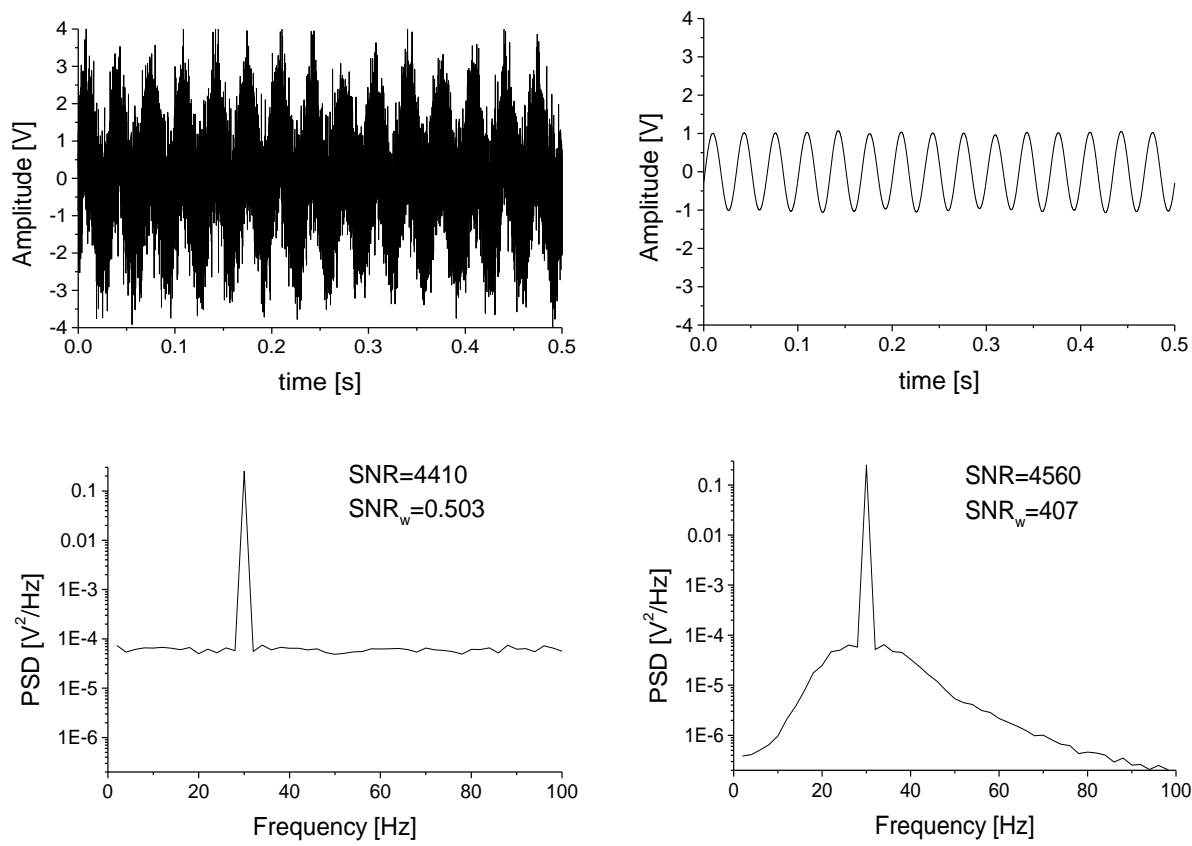

Fig. 3. The plots on the left show $1 \mathrm{~V}$ amplitude $30 \mathrm{~Hz}$ frequency sinusoidal plus $16 \mathrm{kHz}$ bandwidth Gaussian white noise and its power spectral density (PSD). Filtering this signal using a second order Butterworth bandpass filter with cutoff frequencies of $20 \mathrm{~Hz}$ and $40 \mathrm{~Hz}$ results in the signal presented on the right panel. It is easy to see that while the classical $S N R$ is the same within the measurement error, $S N R_{w}$ and the time domain signal reflect the improvement. $S N R$ has a value of 4410 and 4560 while $S N R_{w}$ is obtained as 0.503 and 407 for the original and filtered signal, respectively.

\section{Results}

We carried out our simulations and calculated the input and output SNRs as well as SNR gains for three different values of the amplitude of the deterministic signal $(70 \%, 80 \%$ and $90 \%$ of threshold value) with duty cycles of $10 \%, 20 \%$ and $30 \%$ for each amplitude value. A sample length of 2048 was used for recordings and 1000 samples were averaged to compute the power spectral density and the SNR. The sampling frequency was $8 \mathrm{kHz}$, the frequency of the periodic input signal was set to $31.25 \mathrm{~Hz}$ and the bandwidth of the white noise was $50 \mathrm{kHz}$. We applied both the narrowband and wideband definition of the SNR; the independent variable in each case was the input noise amplitude. Apart from the basic question whether we can obtain SNR gains higher than unity, we examined how the values of the amplitude and duty cycle of the periodic signal influence the SNR and the SNR gain. 

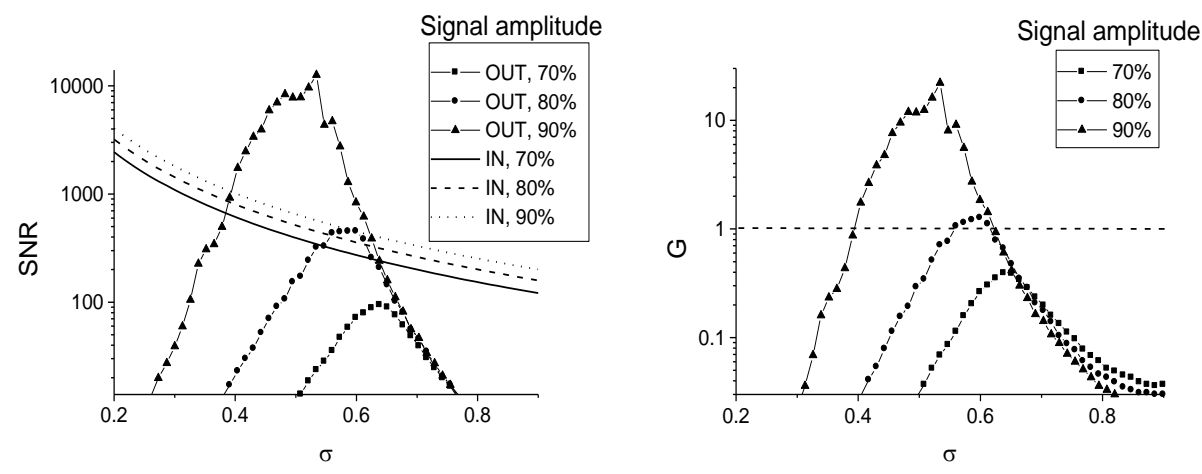

Fig. 4. Input and output SNR and gain as functions of the input noise amplitude. The amplitude of the signal is $70 \%, 80 \%$ and $90 \%$ of the threshold value; the duty cycle is $10 \%$. The amplitude of the noise is denoted by $\sigma$; its value is expressed in units normalized by the threshold.
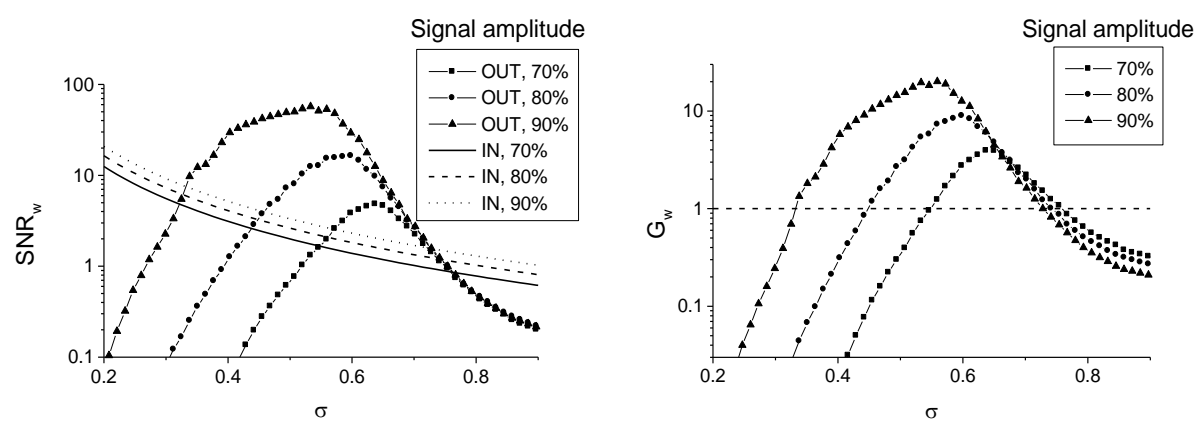

Fig. 5. Wideband input and output SNR and gain as functions of the input noise amplitude. The amplitude of the signal is $70 \%, 80 \%$ and $90 \%$ of the threshold value; the duty cycle is $10 \%$. The amplitude of the noise is denoted by $\sigma$; its value is expressed in units normalized by the threshold.

Figure 4 shows how the narrowband $S N R_{\text {in }}, S N R_{\text {out }}$ and $G$ depend on the input noise intensity for three different amplitudes of the periodic signal. We can conclude two things from this figure. First, SNR improvement is possible in the double-well potential $S N R_{\text {out }}$ can be greater than $S N R_{\text {in }}$ over a certain range of input noise intensity. Second, the value of SNR gain is strongly influenced by the amplitude of the periodic signal, more precisely, higher gains are obtained for larger amplitudes. $G$ exceeds unity only when the amplitude is $80 \%$ or greater, in other words, only in the strongly NLR limit.

Figure 5 is parallel to Fig. 4, only here the wideband quantities are plotted. Comparing these figures to each other, we see how much the SNR definition we use affects the results we get: with the wideband SNR, SNR improvement occurs for all three signal amplitudes and over a wider range of noise intensity than with the narrowband SNR. Figure 6 shows the two types of gains side by side to illustrate the differences between them. 


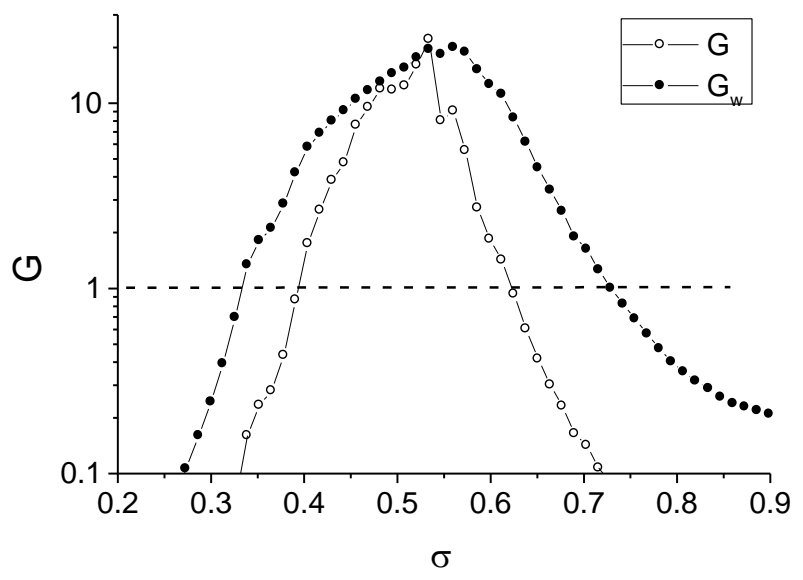

Fig. 6. Narrowband $(G)$ and wideband $\left(G_{w}\right)$ SNR gains compared. The amplitude of the noise is denoted by $\sigma$; its value is expressed in units normalized by the threshold.

In Fig. 7, we can observe how the value of the duty cycle affects the SNR gain, both narrowband and wideband.
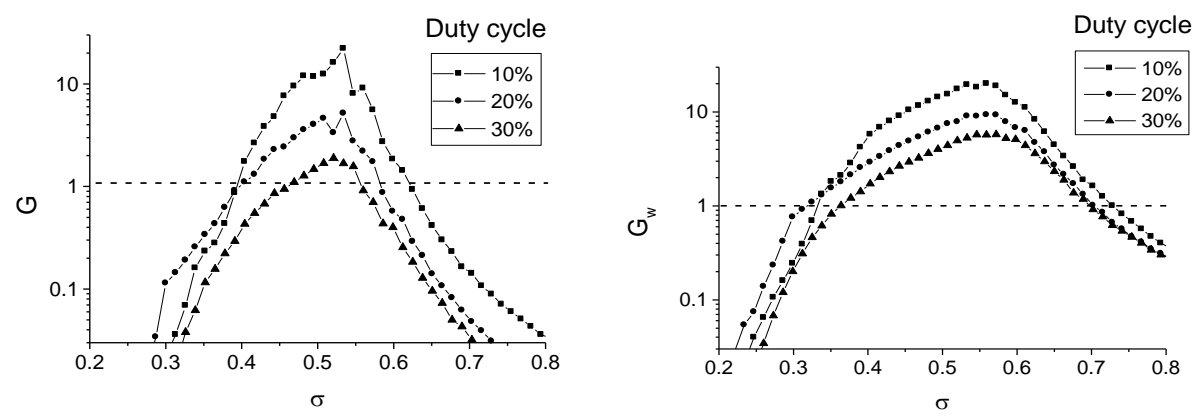

Fig. 7. The plot on the left shows SNR gain versus noise amplitude for different duty cycles of $10 \%, 20 \%$ and $30 \%$. The right panel shows the same with wideband gain. The signal amplitude is $90 \%$ of the threshold value for all curves. The amplitude of the noise is denoted by $\sigma$; its value is expressed in units normalized by the threshold.

It is clear that increasing the duty cycle lowers the SNR gain. One can easily understand this fact: the output signal is similar for different duty cycles, which is reflected in similar $S N R_{\text {out }}$ values, while $S N R_{\text {in }}$ increases with greater duty cycles.

\section{Conclusion}

Using mixed signal simulation techniques, we have shown that one of the most common dynamical SR systems, the double well potential, can provide high SNR amplification if the input excitation is a periodic pulse train plus Gaussian white noise. We have also demonstrated that high SNR gains can be observed for small duty cycles and a signal 
amplitude close to the switching threshold. Note here that our result is not in contradiction with the theoretical result that output SNR cannot exceed input SNR if the system works in the linear response range, since we used large signal amplitude somewhat below the switching threshold, which means that our system worked in the non-linear limit.

We would also like to emphasize that the SNR gain is greater than unity even over a wider range if we apply a much more realistic wideband definition based on the total power of signal and noise.

\section{Acknowledgements}

We thank L. B. Kish for helpful discussions.

\section{References}

[1] L. B. Kiss, Possible breakthrough: significant improvement of signal to noise ratio by stochastic resonance, in Chaotic, Fractal, and Nonlinear Signal Processing, Proc. Am. Institute Phys., ed. R. Katz, Mystic, Connecticut, USA (1996) 382-396.

[2] K. Loerincz, Z. Gingl and L. B. Kiss, A stochastic resonator is able to greatly improve signalto-noise ratio, Phys Lett A 224 (1996) 63-67.

[3] F. Chapeau-Blondeau and X. Godivier, Theory of stochastic resonance in signal transmission by static non-linear systems, Phys Rev E 55 (1997) 1478-1495.

[4] D. G. Luchinsky and P. V. E. McClintock, Irreversibility of classical fluctuations studied in analogue electrical circuits, Nature 389 (1997) 463-466.

[5] S. M. Bezrukov and I. Vodyanoy, Stochastic resonance in non-dynamical systems without response thresholds, Nature 385 (1997) 319-321.

[6] S. Kadar, J. Wang and K. Showalter, Noise-supported traveling waves in sub-excitable media, Nature 391 (1998) 770-773.

[7] S. M. Bezrukov and I. Vodyanoy, Noise-induced enhancement of signal-transduction across voltage-dependent ion channels, Nature 378 (1995) 362-364.

[8] K. Wiesenfeld and F. Moss, Stochastic resonance and the benefits of noise from ice ages to crayfish and squids, Nature 373 (1995) 33-36.

[9] J. M. G. Vilar, G. Gomila and J. M. Rubi, Stochastic resonance in noisy nondynamical systems, Phys Rev Lett 81 (1998) 14-17.

[10] M. E. Inchiosa, A. R. Bulsara, A. D. Hibbs and B. R. Whitecotton, Signal enhancement in a nonlinear transfer characteristic, Phys Rev Lett 80 (1998) 1381-1392.

[11] J. J. Collins, C. C. Chow and T. T. Imhoff, Aperiodic stochastic resonance in excitable systems, Phys Rev E 52 (1995) 3321-3324.

[12] L. B. Kiss, Z. Gingl, Z. Marton, J. Kertesz, F. Moss, G. Schmera and A. R. Bulsara, 1/f noise in systems showing stochastic resonance, J Stat Phys 70 (1993) 451-462.

[13] M. I. Dykman, D. G. Luchinsky, R. Mannella, P. V. E. McClintock, N. D. Stein and N. G. Stocks, Stochastic resonance in perspective, Il Nuovo Cimento D 17D (1995) 661-683.

[14] M. DeWeese and W. Z. Bialek, Information flow in sensory neurons, Il Nuovo Cimento D 17D (1995) 733-741.

[15] Z. Gingl, R. Vajtai and L. B. Kiss, Signal-to-noise ratio gain by stochastic resonance in a bistable system, Chaos, Solitons and Fractals 11 (2000) 1929-1932.

[16] P. Hänggi, M. E. Inchiosa, D. Fogliatti and A. R. Bulsara, Nonlinear stochastic resonance: The saga of anomalous output-input gain, Phys Rev E 62 (2000) 6155-6163.

[17] L. Gammaitoni, P. Hänggi, P. Jung and F. Marchesoni, Stochastic Resonance, Rev Mod Phys 70 (1998) 223-287.

[18] I. Goychuk and P. Hänggi, Stochastic resonance in ion channels characterized by information theory, Phys Rev E 61 (2000) 4272-4280.

[19] L. B. Kish, G. P. Harmer and D. Abbott, Information transfer rate of neurons: stochastic resonance of Shannon's information channel capacity, Fluctuation and Noise Letters 1 (2001) L13-L19. 